\title{
Current Suppression in Transport Through Triple Quantum Dots Coupled to Ferromagnetic Leads
}

\author{
K. WRZEŚNIEWSKI* AND I. WEYMANN \\ Faculty of Physics, Adam Mickiewicz University, Umultowska 85, 61-614 Poznań, Poland
}

\begin{abstract}
We consider transport through triple quantum dot system in a triangular geometry weakly coupled to external ferromagnetic leads. The real-time diagrammatic technique in the lowest order perturbation theory is used to calculate the current and Fano factor in the parallel and antiparallel magnetic configuration of the system as well as the resulting tunnel magnetoresistance (TMR). We focus on the transport regime where the current is suppressed and show that it can lead to negative differential conductance and large super-Poissonian shot noise, which are present in both magnetic configurations. Furthermore, we show that for voltages where the system is trapped in a one-particle dark state the TMR becomes suppressed, while for two-particle blockade, the TMR is much enhanced.
\end{abstract}

DOI: 10.12693/APhysPolA.127.460

PACS: 73.23.-b, 73.21.La, 85.75.-d

\section{Introduction}

Quantum dots are ideal systems to study various correlations between single charges and spins at the nanoscale [1]. They are also very promising for applications in future nanoelectronics, therefore there is a lot of interest in fully understanding their various properties, including electronic transport. In fact, many phenomena known from different areas of physics can be observed and studied in artificial molecules built from several coupled quantum dots. One prominent example, which comes from atomic physics, is related with coherent population trapping in a dark state, which can also occur in triple quantum dot (TQD) systems [2-4]. The presence of such states reveals itself in current suppression and negative differential conductance. Recently, the role of dark states in transport through coherent TQDs weakly coupled to nonmagnetic leads was analyzed by means of the realtime diagrammatic technique [5]. In this paper we will extend these studies by assuming that the external electrodes are made of ferromagnetic materials. Quantum dots, when attached to ferromagnetic leads, can exhibit a considerable tunnel magnetoresistance (TMR) effect when varying the magnetic configuration between parallel and antiparallel one $[6,7]$. The goal is thus to examine the magnetoresistive properties of TQDs and the effect of spin-dependent tunneling on transport through TQDs with a focus on transport regimes where current suppression occurs.

\section{Theoretical description}

The system consists of three single-level quantum dots in a triangular geometry, coupled to each other via hopping parameter $t$ and attached to external ferromagnetic

${ }^{*}$ corresponding author; e-mail: kacper.wrzesniewski@gmail.com leads, see Fig. 1. The first (second) dot is coupled to the left (right) lead with coupling strength $\Gamma^{\sigma}$ and the bias voltage is applied symmetrically to the system. The magnetizations of the leads are assumed to form either parallel or antiparallel magnetic configuration. The Hamiltonian of system is given by, $H=H_{\text {Leads }}+H_{\mathrm{TQD}}+H_{\mathrm{T}}$, with $H_{\text {Leads }}$ describing noninteracting electrons in the leads, $H_{\text {Leads }}=\sum_{j=L, R} \sum_{k \sigma} \varepsilon_{j k \sigma} c_{j k \sigma}^{\dagger} c_{j k \sigma}$, where $c_{j k \sigma}^{\dagger},\left(c_{j k \sigma}\right)$ is the creation (annihilation) operator of an electron with spin $\sigma$ momentum $k$ in the left $(j=L)$ and right $(j=R)$ lead, while $\varepsilon_{j k \sigma}$ denotes the corresponding single-particle energy. The TQD Hamiltonian reads

$$
\begin{aligned}
& H_{\mathrm{TQD}}=\sum_{j \sigma} \varepsilon_{j} n_{j \sigma}+U \sum_{j} n_{j \uparrow} n_{j \downarrow}+\frac{U^{\prime}}{2} \sum_{\langle i j\rangle} \sum_{\sigma \sigma^{\prime}} n_{\mathrm{i} \sigma} n_{\mathrm{j} \sigma^{\prime}} \\
& \quad+\sum_{\langle i j\rangle} \frac{t}{2} \sum_{\sigma}\left(d_{i \sigma}^{\dagger} d_{j \sigma}+d_{j \sigma}^{\dagger} d_{i \sigma}\right) .
\end{aligned}
$$

Here, $n_{j \sigma}=d_{j \sigma}^{\dagger} d_{j \sigma}$, where $d_{j \sigma}^{\dagger}$ creates an electron with spin $\sigma$ in the $j$ th quantum dot, while $\varepsilon_{j}$ denotes the respective energy. The second and third terms take into account the on-site Coulomb interaction $U$ and the interdot Coulomb correlation denoted by $U^{\prime}$, respectively. The last term of $H_{\mathrm{TQD}}$ describes the hopping between the dots with $t$ being the hopping parameter, assumed equal for all dots. The summation $\langle i j\rangle$ is over nearestneighbor dots and the parameters $U^{\prime}$ and $t$ are divided by 2 to avoid double counting. Finally, the tunneling Hamiltonian is given by

$$
H_{\mathrm{T}}=\sum_{j=L, R} \sum_{k \sigma}\left(v_{j} c_{j k \sigma}^{\dagger} d_{j k \sigma}+\text { H.c. }\right),
$$

where $v_{j}$ denotes the tunnel matrix elements between the $j$ th lead and the corresponding dot, see Fig. 1. The respective coupling strength is given by, $\Gamma_{j}^{\sigma}=2 \pi\left|t_{j}\right| \rho_{j}^{\sigma}$, where $\rho_{j}^{\sigma}$ is the spin-dependent density of states in lead $j$. With the definition of spin polarization for lead $j, p_{j}=\left(\rho_{j}^{+}-\rho_{j}^{-}\right) /\left(\rho_{j}^{+}+\rho_{j}^{-}\right)$, the coupling becomes, $\Gamma_{j}^{+(-)}=\Gamma_{j}\left(1 \pm p_{j}\right)$, where $\Gamma_{j}=\left(\Gamma_{j}^{+}+\Gamma_{j}^{-}\right) / 2$. Here, 
$\Gamma_{j}^{+}\left(\Gamma_{j}^{-}\right)$stands for coupling between given dot to the spin-majority (spin-minority) electron band of lead $j$. In the following we assume, $\Gamma_{L}=\Gamma_{R} \equiv \Gamma$ and $p_{L}=p_{R} \equiv p$. We also take the limit of $U \rightarrow \infty$ and exclude doubly occupied state of each dot from calculations.

To determine the transport characteristics we use the real-time diagrammatic technique $[8,9]$. This method is based on a systematic perturbation expansion of the reduced density matrix and the operators of interest with respect to the coupling strength $\Gamma$. In this analysis we assume that the coupling is very weak and consider only the lowest order of expansion, which corresponds to sequential tunneling. Moreover, we also assume that the hopping between the dots is relatively large, $t \gg \Gamma$. This leads to significant overlap of the wave functions of the neighboring dots, resulting in the formation of molecular states, $|\chi\rangle$, through which transport takes place.

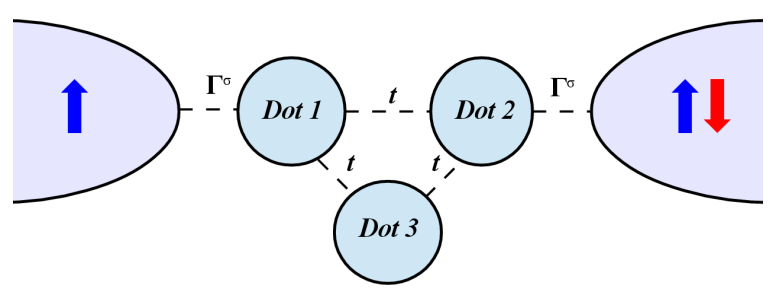

Fig. 1. Schematic of TQD in a triangular geometry. Dot 1 (2) is coupled to the left (right) lead with coupling strength $\Gamma^{\sigma}$. Each dot is coupled to remaining two dots with hopping parameter $t$.

Within the real-time diagrammatic technique, the occupation probabilities $p_{\chi}$ can be found from a master-like equation $[8,9]$. On the other hand, the current can be calculated from $[8,9], I=(e / 2 \hbar) \operatorname{Tr}\left\{\boldsymbol{W}^{I} \boldsymbol{p}\right\}$, where $\boldsymbol{W}^{I}$ is the self-energy matrix which takes into account the number of particles transferred through the system and $\boldsymbol{p}$ is the probability vector. In the following, we study the bias dependence of the current in the parallel $\left(I^{\mathrm{P}}\right)$ and antiparallel $\left(I^{\mathrm{AP}}\right)$ magnetic configuration and the TMR, defined as [7], $\mathrm{TMR}=I^{\mathrm{P}} / I^{\mathrm{AP}}-1$. Moreover, we also analyze the Fano factor, $F=S /(2 e|I|)$, where $S$ denotes the shot noise. The formula for $S$ can be found in Ref. [9].

\section{Results and conclusions}

The currents $I^{\mathrm{P}}$ and $I^{\mathrm{AP}}$ as well as the resulting TMR are shown in Fig. 2a for $\varepsilon_{1}=\varepsilon_{3} \equiv \varepsilon=1 \mathrm{meV}$ and $\varepsilon-\varepsilon_{2} \equiv \delta=0.5 \mathrm{meV}$. For this level configuration, when $1 / 2 \lesssim e V / U^{\prime} \lesssim 3 / 2$, the system is trapped in a oneparticle dark state. The TQD is then in a state, in which the wave function is extended over only two dots, one of which is not directly coupled to the leads. As a result, the transfer of electrons through the system is blocked. Although in the dark state regime the current is suppressed, due to nonzero temperature there is still a finite occupation probability of single-particle states, which are

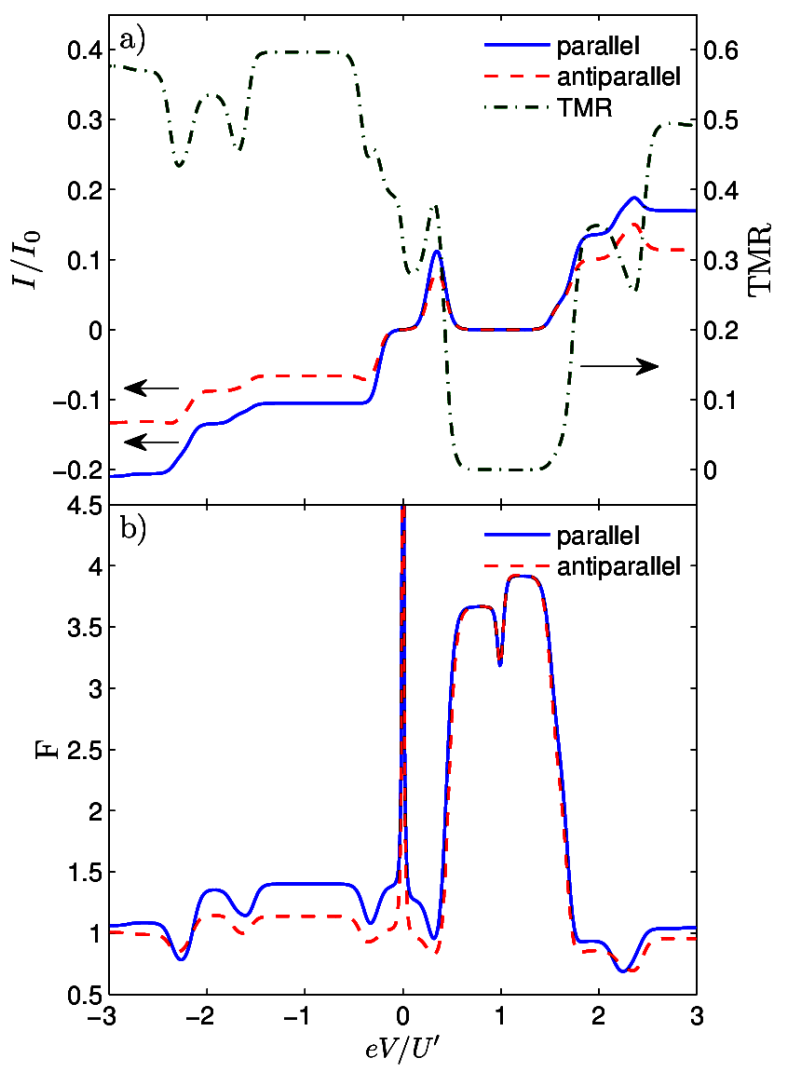

Fig. 2. The bias voltage dependence of (a) the current in the parallel and antiparallel configuration and the resulting TMR, and (b) the Fano factor in both magnetic configurations. The parameters are as follows: $U^{\prime}=$ $10 \mathrm{meV}, \varepsilon_{1}=\varepsilon_{3} \equiv \varepsilon=1 \mathrm{meV}, \varepsilon-\varepsilon_{2} \equiv \delta=0.5 \mathrm{meV}$, $t=-1 \mathrm{meV}, \Gamma=0.025 \mathrm{meV}, p=0.5, T=0.2 \mathrm{meV}$ and $I_{0}=e \Gamma / \hbar$.

extended over all three dots and thus contribute to transport. This gives rise to a thermally-activated nonzero current and to the TMR in the blockade regime. As can be seen in Fig. 2a, for negative bias voltage both currents $I^{\mathrm{P}}$ and $I^{\mathrm{AP}}$ display typical Coulomb staircase dependence. However, for positive bias, the current first increases to drop when the system becomes trapped in a dark state, which happens for $1 / 2 \lesssim e V / U^{\prime} \lesssim 3 / 2$. The system exhibits then negative differential conductance, irrespective of magnetic configuration. Moreover, $I^{\mathrm{P}}>I^{\mathrm{AP}}$, in the whole range of considered bias voltage, except for $1 / 2 \lesssim e V / U^{\prime} \lesssim 3 / 2$, when one finds $I^{\mathrm{P}} \approx I^{\mathrm{AP}}$. As a result, the TMR is always positive and reaches approximately zero, when the system is trapped in oneparticle dark state, see Fig. 2a.

The presence of dark states is also revealed in the dependence of the Fano factor on the bias voltage. The Fano factor describes the deviation of the shot noise from the Poissonian noise for uncorrelated events. In the dark state regime, for both parallel and antiparallel configuration, the shot noise becomes super-Poissonian with 
Fano factor much larger than unity, see Fig. 2b. For $e V \rightarrow 0$, the Fano factor diverges due to the fact that $I \rightarrow 0$, while $S$ is dominated by thermal noise. We also note that generally $F^{\mathrm{P}}>F^{\mathrm{AP}}$, this results from the fact that in the parallel configuration there is a larger imbalance between spin-resolved transport channels compared to the antiparallel configuration, which enhances the fluctuations of the current.

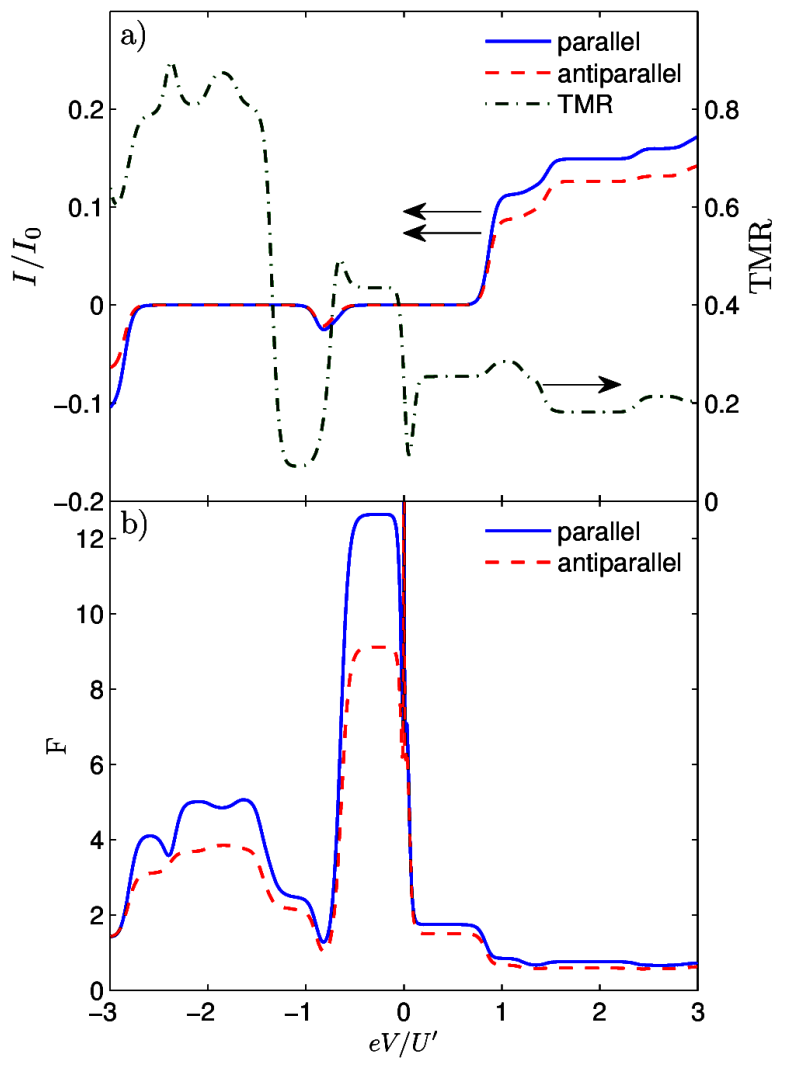

Fig. 3. The bias voltage dependence of (a) the current in the parallel and antiparallel configuration and the resulting TMR, and (b) the Fano factor in both magnetic configurations. The parameters are the same as in Fig. 2 with $\varepsilon=-25 \mathrm{meV}$.

By lowering the levels of the dots, the TQD becomes successively occupied with electrons. The transport characteristics for the case when there are three electrons in the TQD for $V=0$ are shown in Fig. 3. Now, for positive bias the current grows monotonically and shows Coulomb steps, while for negative bias voltage one observes current suppression and negative differential conductance. With increasing negative bias, the current exhibits a small peak for $e V / U^{\prime} \approx-0.8$, i.e. when the applied bias matches the excitation energy between three and two-particle states. However, for $e V / U^{\prime}<-0.8$, the system becomes trapped in a two-particle blockade and the current is suppressed in a wide range of negative bias voltage, see Fig. 3a. In this blockade, the occupancy of the second dot is one, while the occupancies of first and third dots are equal to one half. Because of large on-dot Coulomb interaction, the electrons cannot tunnel from right lead to the second dot and transport is then blocked. Note, that electronic spacial distribution in this blockade is significantly different compared to single-particle dark state where clearly one of the dots was not occupied at all. Now, its occupancy is equal to one, forbidding tunneling from the lead to this dot due to large $U$. This blockade is thus rather due to finite Coulomb correlations in the dots, contrary to one-particle blockade discussed above, which was due to the trapping in a dark state.

The magnetoresistive properties of the system are also different than those in the case shown in Fig. 2. For two-particle blockade, the TMR is greatly enhanced, see Fig. 3a. This can be attributed to nonequilibrium spin accumulation in triplet states that develops in the antiparallel configuration, leading to large difference between the currents $I^{\mathrm{P}}$ and $I^{\mathrm{AP}}$. Moreover, in the blockade regimes due to Coulomb interactions, we find superPoissonian shot noise, which is enhanced in the parallel configuration compared to the antiparallel one, see Fig. 3b.

In conclusion, we have studied the spin-resolved sequential transport through triple quantum dot in triangular geometry weakly coupled to ferromagnetic leads. We showed that when the system is trapped in one-particle dark state, the TMR is generally suppressed, while for two-particle blockade we predicted an enhancement of TMR. Both blockade regimes are also associated with super-Poissonian shot noise, which is larger in the parallel configuration compared to the antiparallel one.

\section{Acknowledgments}

This work was supported by the National Science Center in Poland as the Project No. DEC2013/10/E/ST3/00213.

\section{References}

[1] L.P. Kouwenhoven, D.G. Austing, S. Tarucha, Rep. Prog. Phys. 64, 701 (2001).

[2] C. Emary, Phys. Rev. B 76, 245319 (2007).

[3] C. Poltl, C. Emary, T. Brandes, Phys. Rev. B 80, 115313 (2009).

[4] T. Kostyrko, B.R. Bułka, Phys. Rev. B 79, 075310 (2009).

[5] I. Weymann, B. R. Bułka, J. Barnaś, Phys. Rev. B 83, 195302 (2011).

[6] I. Weymann, J. König, J. Martinek, J. Barnaś, G. Schön, Phys. Rev. B 72, 115334 (2005).

[7] J. Barnaś, I. Weymann, J. Phys.: Condens. Matter 20, 423202 (2008).

[8] H. Schoeller, G. Schön, Phys. Rev. B 50, 18436 (1994); J. König, J. Schmid, H. Schoeller, G. Schön, Phys. Rev. B 54, 16820 (1996).

[9] A. Thielmann, M. H. Hettler, J. König, G. Schön, Phys. Rev. Lett. 95, 146806 (2005). 